\title{
Interval Type-2 Intuitionistic Fuzzy Logic System for Non-linear System Prediction
}

\author{
Imo Eyoh, Student Member, IEEE ${ }^{1,2}$, Robert John, Senior Member, IEEE ${ }^{1,2}$ and Geert De Maere 2 \\ ${ }^{1}$ Laboratory for Uncertainty in Data and Decision Making (LUCID) \\ ${ }^{2}$ Automated Scheduling, Optimisation and Planning (ASAP) \\ School of Computer Science \\ University of Nottingham, UK \\ Email: (ije, rij,gdm)@cs.nott.ac.uk
}

\begin{abstract}
This paper presents an approach to prediction based on a new interval type-2 intuitionistic fuzzy logic system (IT2IFLS) of Takagi-Sugeno-Kang (TSK) fuzzy inference. The gradient descent algorithm (GDA) is used to adapt the parameters of the IT2IFLS. The empirical comparison is made on the designed system using two synthetic datasets. Analysis of our results reveal that the presence of additional degrees of freedom in terms of non-membership functions and hesitation indexes in IT2IFLS tend to reduce the root mean square error (RMSE) of the system compared to a type-1 fuzzy logic approach and some interval type-2 fuzzy systems.
\end{abstract}

\section{INTRODUCTION}

$\mathrm{F}$ UZZY set (FS) theory was introduced by Zadeh [1] as a generalisation of the classical notion of a set and a proposition to accommodate fuzziness (degree of truth) as expressed in natural or human language [2], [3]. The attraction of FS lie in the resulting model's capacity to represent nonlinear input/output relationships using qualitative IF . . THEN rules [3]. Despite the extensive use of type-1 FS (T1FS) and its connotation of uncertainty, previous works have established that T1 fuzzy logic models uncertainty to a certain degree in many applications and may not handle or minimise the effects of uncertainties inherent in some real world applications [4]. To address this problem, Zadeh [5] proposed an extension of his previous T1FS theory to include type-2 fuzzy set (T2FS) theory capable of handling uncertainties that T1 struggles with because membership grades of T2FS are themselves fuzzy.

Atanassov [6] extended the concept of Zadeh's fuzzy sets to intuitionistic fuzzy sets (IFSs), which deal with uncertainty by considering both the degrees of membership and nonmembership of an element $x$ to a fuzzy set $A$ with some degree of hesitancy. According to Olej and Hajek [7], the representation of attributes by means of membership and nonmembership functions provides a better way to express uncertainty. Castillo et al [8] lend credence to this when they opined that the presence of non-membership or hesitation index gives more possibility to represent imperfect knowledge and to adequately describe many real world problems. Atanassov and Gargov [9] extended the concept of IFS to interval valued intuitionistic fuzzy sets (IVIFS) which are characterised by membership and non-membership functions and defined in the interval $[0,1]$. The resulting T2 fuzzy logic systems (FLSs) whose degrees of membership are intervals are capable of providing better performance in some applications than the T1FLSs [10], [11].

To the knowledge of the authors, there is currently no work in the literature where interval type-2 intuitionistic fuzzy set (IT2IFS) is applied in a fuzzy logic inference system. Our motivation is to apply IT2IFS to model uncertainty in data. In this study, an IT2IFLS based on TSK fuzzy inference system (FIS) is presented and the learning algorithms for the adaptation of its parameters are derived.

The rest of the paper is structured as follows: In section II, IFS, T2IFS and IT2IFS are defined. In section III, IT2IFLS is designed and parameter update rules are derived. We present our results in Section IV, and conclude in section V.

\section{Type-1 And Type-2 IntUitionistic FuZZy SET}

\section{A. Intuitionistic Fuzzy Set (IFS)}

Definition 1. [6] Given a non-empty set $X$, an intuitionistic fuzzy set $A^{*}$ in $X$ is an object having the form: $A^{*}=$ $\left.\left\{\left(x, \mu_{A^{*}}(x), \nu_{A^{*}}(x)\right): x \in X\right)\right\}$, where the function $\mu_{A^{*}}(x)$ $: X \rightarrow[0,1]$ defines the degree of membership and $\nu_{A^{*}}(x)$ : $X \rightarrow[0,1]$ defines the degree of non-membership of element $x \in X$ and for every element $x \in X, 0 \leq \mu_{A^{*}}(x)+\nu_{A^{*}}(x) \leq$ 1 .

When $\nu_{A^{*}}(x)=1-\mu_{A^{*}}(x)$ for every $x \in X$, then the set $A$ is a fuzzy set. Moreover, Atanassov also specified an hesitation degree, $\pi$, defined as 1 minus the sum of the degree of membership and non-membership of an element to a set. That is, $\pi_{A^{*}}(x)=1-\left(\mu_{A^{*}}(x)+\nu_{A^{*}}(x)\right)$. This is called the hesitancy factor or intuitionistic fuzzy index of $x$ in $A^{*}$.

\section{B. Type-2 Intuitionistic Fuzzy Set (T2IFS)}

Here, we provide, for the first time, a new definition for a T2IFS. A T2IFS $\tilde{A}^{*}$ in the universe of discourse, $X$ consists of type-2 membership and non-membership grades of $x \in X$ defined as $\mu_{\tilde{A}^{*}}(x, u): u \in J_{x}^{\mu} \subseteq[0,1]$ and $\nu_{\tilde{A}^{*}}(x, u): u \in$ $J_{x}^{\nu} \subseteq[0,1]$ respectively. The primary membership $\left(J_{x}^{\mu}\right)$ and primary non-membership $\left(J_{x}^{\nu}\right)$ of element $x \in \tilde{A}^{*}$ are elements in the domain $(x, u)$ which form supports of T2IFS in the third 
dimension for membership and non-membership respectively and are defined as follows:

$$
\begin{gathered}
J_{x}^{\mu}=\left\{(x, u): u \in\left[\underline{\mu}_{\tilde{A}^{*}}(x), \bar{\mu}_{\tilde{A}^{*}}(x)\right]\right\} \\
J_{x}^{\nu}=\left\{(x, u): u \in\left[\underline{\nu}_{\tilde{A}^{*}}(x), \bar{\nu}_{\tilde{A^{*}}}(x)\right]\right\}
\end{gathered}
$$

Definition 2. A T2IFS denoted by $\tilde{A}^{*}$ is characterised by a type-2 membership function $\mu_{\tilde{A}^{*}}(x, u)$, and a type-2 nonmembership function $\nu_{\tilde{A}^{*}}(x, u)$, i.e.,

$$
\begin{aligned}
\tilde{A}^{*}=\left\{(x, u), \mu_{\tilde{A^{*}}}(x, u), \nu_{\tilde{A}^{*}}(x, u)\right. & \mid \forall x \in X, \\
& \left.\forall u \in J_{x}^{\mu}, \forall u \in J_{x}^{\nu}\right\}
\end{aligned}
$$

in which $0 \leq \mu_{\tilde{A}^{*}}(x, u) \leq 1$ and $0 \leq \nu_{\tilde{A}^{*}}(x, u) \leq 1$ where $\forall u \in J_{x}^{\mu}$ and $\forall u \in J_{x}^{\nu}$ conform to the T1 constraint that $0 \leq \mu_{A^{*}}(x)+\nu_{A^{*}}(x) \leq 1$. That is, when uncertainties disappear, a T2 membership and non-membership functions must reduce to a T1 membership and non-membership functions respectively. Also the amplitudes of both membership and non-membership functions must lie in the closed interval of 0 and 1 . That is, $0 \leq \mu_{\tilde{A}^{*}}(x, u) \leq 1$ and $0 \leq \nu_{\tilde{A}^{*}}(x, u) \leq$ 1. Alternatively,

$$
\int_{x \in X}\left[\int_{u \in J_{x}^{\mu}} \int_{u \in J_{x}^{\nu}}\left\{\mu_{\tilde{A^{*}}}(x, u), \nu_{\tilde{A}^{*}}(x, u)\right\}\right] /(x, u)
$$

where $\iiint$ represents union over all admissible values of $x$ and $u$ for the membership and non-membership over a continuous universe of discourse, and $\int$ is replaced by $\sum$ for discrete universe of discourse. When the secondary membership functions $\mu_{\tilde{A}^{*}}(x, u)=1$, and secondary non-membership functions $\nu_{\tilde{A}^{*}}(x, u)=1$, a T2IFS translates to an IT2IFS (see Figure 1 and Equation (1)).

Definition 3. [12] An IT2IFS, $\tilde{A}^{*}$, is characterised by membership bounding functions and non-membership bounding functions defined as $\bar{\mu}_{\tilde{A}^{*}}(x), \underline{\mu}_{\tilde{A}^{*}}(x)$ and $\bar{\nu}_{\tilde{A}^{*}}(x), \underline{\nu}_{\tilde{A}^{*}}(x)$ respectively for all $x \in X$ with constraints: $0 \leq \bar{\mu}_{\tilde{A}^{*}}(x)+$ $\underline{\nu}_{\tilde{A}^{*}}(x) \leq 1$ and $0 \leq \underline{\mu}_{\tilde{A}^{*}}(x)+\bar{\nu}_{\tilde{A}^{*}}(x) \leq 1$.

For each $x \in X$, there exist a third tuple $\pi(x)$ called the IF-index or hesitancy degree which comes as a result of an expert not being certain of the degree of membership and nonmembership of element $x \in X$.

Two IF-indexes used in this study are the IF-index of center and IF-index of variance previously used in [13] and defined in this work as:

$$
\begin{gathered}
\pi_{c}(x)=\max \left(0,\left(1-\left(\mu_{\tilde{A^{*}}}(x)+\nu_{\tilde{A^{*}}}(x)\right)\right)\right) \\
\bar{\pi}_{v a r}(x)=\max \left(0,\left(1-\left(\bar{\mu}_{\tilde{A^{*}}}(x)+\underline{\nu}_{\tilde{A^{*}}}(x)\right)\right)\right) \\
\underline{\pi}_{v a r}(x)=\max \left(0,\left(1-\left(\underline{\mu}_{\tilde{A^{*}}}(x)+\bar{\nu}_{\tilde{A^{*}}}(x)\right)\right)\right)
\end{gathered}
$$

such that: $0 \leq \pi_{c}(x) \leq 1$ and $0 \leq \pi_{\text {var }}(x) \leq 1$.

As defined above, an IT2IFS $\tilde{A}^{*}$ is characterised by interval type-2 membership function, $\mu_{\tilde{A}^{*}}(x, u)$ and interval type-2 non-membership function, $\nu_{\tilde{A}^{*}}(x, u)$ for all $x \in X$ expressed as:

$$
\begin{aligned}
& \tilde{A}^{*}=\int_{x \in X} \int_{u \epsilon J_{x}^{\mu}} \int_{u \epsilon J_{x}^{\nu}} 1 /(x, u) \\
& =\int_{x \in X}\left[\int_{u \epsilon J_{x}^{\mu}} \int_{u \epsilon J_{x}^{\nu}} 1 /(u)\right] / x
\end{aligned}
$$

where $x$ is the primary variable, and $u$ is the secondary variable. The uncertainty about an IT2IFS is completely described by the footprints of uncertainty (FOUs) that are bounded by two T1 membership functions - an upper membership function (UMF) given as $\bar{\mu}_{\tilde{A}^{*}}(x)$ and lower membership function (LMF) expressed as $\underline{\mu}_{\tilde{A}^{*}}(x)$ and two T1 non-membership functions which are - the upper non-membership function (UNMF), $\bar{\nu}_{\tilde{A}^{*}}(x)$ and the lower non-membership function (LNMF), $\underline{\nu}_{\tilde{A}^{*}}(x)$ as illustrated in Figure 1. The uncertainty about the IT2IFS is conveyed by the combination of both membership and non-membership functions into membership and non-membership FOUs (see Figure (1)) and defined as follows:

$$
\begin{gathered}
\operatorname{FOU}_{\mu}\left(\tilde{A}^{*}\right)=\bigcup_{\forall x \in X}\left[\underline{\mu}_{\tilde{A^{*}}}(x), \bar{\mu}_{\tilde{A}^{*}}(x)\right] \\
\operatorname{FOU}_{\nu}\left(\tilde{A}^{*}\right)=\bigcup_{\forall x \in X}\left[\underline{\nu}_{\tilde{A^{*}}}(x), \bar{\nu}_{\tilde{A}^{*}}(x)\right]
\end{gathered}
$$

From Equations (2) and (3), the FOU of IT2IFS can be expressed by the union of all the primary memberships and primary non-memberships.

$$
\operatorname{FOU}\left(\tilde{A}^{*}\right)=\bigcup_{\forall x \in X}\left[\underline{\mu}_{\tilde{A^{*}}}(x), \bar{\mu}_{\tilde{A}^{*}}(x), \underline{\nu}_{\tilde{A}^{*}}(x), \bar{\nu}_{\tilde{A}^{*}}(x)\right]
$$

\section{INTERVAL TYPE-2 INTUITIONISTIC FUZZY LOGIC SYSTEM}

Similar to a type-2 fuzzy logic system (T2FLS), a T2IFLS consists of the fuzzifier, rule base, fuzzy inference engine and output processing module but because of the intuitionism involved in the fuzzy set, we shall refer to the T2IFLS modules as intuitionistic fuzzifier, intuitionistic rule base, intuitionistic fuzzy inference engine and intuitionistic output processor.

\section{A. Fuzzification}

The fuzzification process involves the mapping of a numeric input vector $x \in X$ into an IT2IFS $\tilde{A}^{*}$ in $\mathrm{X}$ which activates the inference engine.

In this study, intuitionistic Gaussian membership and nonmembership functions with uncertain standard deviation are utilised which are defined as follows.

$$
\begin{aligned}
& \overline{\mu_{i k}}\left(x_{i}\right)=\exp \left(-\frac{\left(x_{i}-c_{i k}\right)^{2}}{2 \bar{\sigma}_{2, i k}^{2}}\right) *\left(1-\pi_{c, i k}\left(x_{i}\right)\right) \\
& \underline{\mu_{i k}}\left(x_{i}\right)=\exp \left(-\frac{\left(x_{i}-c_{i k}\right)^{2}}{2 \sigma_{1, i k}^{2}}\right) *\left(1-\pi_{c, i k}\left(x_{i}\right)\right)
\end{aligned}
$$




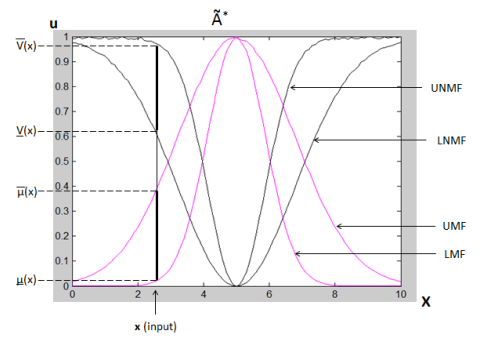

Fig. 1: An IT2 intuitionistic Gaussian membership and nonmembership functions - IT2IFS

$$
\begin{array}{r}
\overline{\nu_{i k}}\left(x_{i}\right)=\left(1-\bar{\pi}_{\text {var }, i k}\left(x_{i}\right)\right)-\left[\exp \left(-\frac{\left(x_{i}-c_{i k}\right)^{2}}{2 \bar{\sigma}_{1, i k}^{2}}\right)\right. \\
\left.*\left(1-\pi_{c, i k}\left(x_{i}\right)\right)\right] \\
\underline{\nu_{i k}}\left(x_{i}\right)=\left(1-\underline{\pi}_{v a r, i k}\left(x_{i}\right)\right)-\left[\exp \left(-\frac{\left(x_{i}-c_{i k}\right)^{2}}{2 \underline{\sigma}_{2, i k}^{2}}\right)\right. \\
\left.*\left(1-\pi_{c, i k}\left(x_{i}\right)\right)\right]
\end{array}
$$

where $\pi_{c, i k}$ is the IF-index of center and $\pi_{v a r, i k}$ is the IF-index of variance. The parameters $\bar{\sigma}_{2, i k}, \underline{\sigma}_{1, i k}, \pi_{c, i k}$ and $\pi_{v a r, i k}$ are premise parameters that define the degree of membership and non-membership of each element to the fuzzy set $\tilde{A}^{*}$. They jointly define the FOUs of the IT2IFS.

\section{B. Rules}

The IF-THEN rule of an IT2IFLS can be expressed as follows:

$$
\begin{gathered}
R_{k}: I F x_{1} \text { is } \tilde{A}^{*}{ }_{1 k} \text { and } x_{2} \text { is } \tilde{A}^{*}{ }_{2 k} \text { and } \cdots \text { and } x_{n} \text { is } \tilde{A}^{*}{ }_{n k} \\
\text { THEN } y_{k} \text { is } f\left(x_{1}, x_{2}, \cdots, x_{n}\right) \\
=w_{1 k} x_{1}+w_{2 k} x_{2}+\cdots+w_{n k} x_{n}+b_{k}
\end{gathered}
$$

where $\tilde{A}^{*}{ }_{1 k}, \tilde{A}^{*}{ }_{2 k}, \cdots, \tilde{A}^{*}{ }_{i k}, \cdots, \tilde{A}^{*}{ }_{n k}$ are IT2IFS and $y_{k}$ is the output of the kth rule formed by linear combination of the input vector: $\left(x_{1}, x_{2}, \cdots, x_{n}\right)$. The rule representation of IT2IFLS is similar to the classical IT2FLS, the only exception is that both membership and non-membership functions are involved in the output of the IT2IFLS.

\section{Inference}

There are generally two main types of fuzzy inferencing namely: Mamdani and TSK which differ in their representation and output evaluation. In this work, we assume a TSK fuzzy inferencing where the output of each IF-THEN rule is a linear function. There are basically three models for generating the output of a type-2 TSK inference system namely [14]:

- Model I: The antecedent parts are type-2 fuzzy set while the consequent parts are type 1 fuzzy sets denoted by A2-C1.

- Model II: The antecedent parts are type-2 fuzzy sets with crisp numbers as consequents denoted by A2-C0.

- Model III: Both the antecedent and consequent parts are T1 fuzzy sets represented as A1-C1.
In this study, model II is adopted to investigate the reasoning behind IT2IFLS with learning ability similar to adaptiveneural network-based fuzzy inference system (ANFIS) [15] and T2-ANFIS [16] approaches. In our study, the antecedent parts are IT2IFS while the consequent parts are crisp values. An IT2IFLS structure with two inputs, three membership functions and nine rules is as shown in Figure 2. According

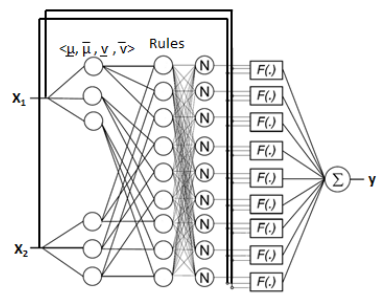

Fig. 2: An IT2IFLS Structure - adapted from [17]

to [18], the output of IFIS-TSK can be computed using two approaches: (i) by the composition of membership output, $y^{\mu}$ and non-membership output functions $y^{\nu}$ (ii) by direct defuzzification. In this study, the former approach is adopted and the output of IT2IFLS is defined as follows:

$y=\frac{(1-\beta) \sum_{k=1}^{M}\left(\underline{f_{k}^{\mu}}+\overline{f_{k}^{\mu}}\right) y_{k}^{\mu}}{\sum_{k=1}^{M} \underline{f_{k}^{\mu}}+\sum_{k=1}^{M} \overline{f_{k}^{\mu}}}+\frac{\beta \sum_{k=1}^{M}\left(\underline{f_{k}^{\nu}}+\overline{f_{k}^{\nu}}\right) y_{k}^{\nu}}{\sum_{k=1}^{M} \underline{f_{k}^{\nu}}+\sum_{k=1}^{M} \overline{f_{k}^{\nu}}}$

where $\underline{f}_{k}^{\mu}, \bar{f}_{k}^{\mu}, \underline{f}_{k}^{\nu}$ and $\bar{f}_{k}^{\nu}$ are the lower membership, upper membership, lower non-membership and upper nonmembership firing strength respectively. In this study, the implication operator employed is the "prod" $t$-norm such that:

$$
\begin{aligned}
& \underline{f_{k}^{\mu}}(x)=\underline{\mu}_{\tilde{A}^{*} 1 k}\left(x_{1}\right) * \underline{\mu}_{\tilde{A}^{*}{ }_{2 k}}\left(x_{2}\right) * \cdots * \underline{\mu}_{\tilde{A}^{*}{ }_{n k}}\left(x_{n}\right) \\
& \overline{f_{k}^{\mu}}(x)=\bar{\mu}_{\tilde{A}^{*}{ }_{1 k}}\left(x_{1}\right) * \bar{\mu}_{\tilde{A}^{*}{ }_{2 k}}\left(x_{2}\right) * \cdots * \bar{\mu}_{\tilde{A}^{*}{ }_{n k}}\left(x_{n}\right) \\
& \underline{f_{k}^{\nu}}(x)=\underline{\nu}_{\tilde{A}^{*} 1 k}\left(x_{1}\right) * \underline{\nu}_{\tilde{A}^{*}{ }_{2 k}}\left(x_{2}\right) * \cdots * \underline{\nu}_{\tilde{A}^{*}{ }_{n k}}\left(x_{n}\right) \\
& \overline{f_{k}^{\nu}}(x)=\bar{\nu}_{\tilde{A}^{*}{ }_{1 k}}\left(x_{1}\right) * \bar{\nu}_{\tilde{A}^{*}{ }_{2 k}}\left(x_{2}\right) * \cdots * \bar{\nu}_{\tilde{A}^{*}{ }_{n k}}\left(x_{n}\right)
\end{aligned}
$$

where $*$ is the "prod" operator, $y_{k}^{\mu}$ and $y_{k}^{\nu}$ are the output of the $k$ th rule corresponding to membership and non-membership function respectively. In IT2IFLS-TSK, the final output is a weighted average of each IF-THEN rule's output and as such do not require any defuzzification procedure [19]. The parameter $\beta$ is a user defined parameter, $0 \leq \beta \leq 1$; specifying the contribution of the membership and non-membership values in the final output. Obviously, if $\beta=0$, the outputs of the IT2IFLS is determined by the membership function and if $\beta=1$, then only the non-membership will contribute to the system's outputs. This study utilizes gradient descent algorithm (GDA) for the update of both the antecedent and the consequent parts of the rules. The cost function for a single output is defined as:

$$
E=\frac{1}{2}\left(y^{a}-y\right)^{2}
$$


where $y^{a}$ is the actual output and $y$ is the network output. The parameter update rules are as follows:

$$
\begin{gathered}
w_{i k}(t+1)=w_{i k}(t)-\gamma \frac{\partial E}{\partial w_{i k}} \\
b_{k}(t+1)=b_{k}(t)-\gamma \frac{\partial E}{\partial b_{k}} \\
c_{i k}(t+1)=c_{i k}(t)-\gamma \frac{\partial E}{\partial c_{i k}} \\
\sigma_{1, i k}(t+1)=\sigma_{1, i k}(t)-\gamma \frac{\partial E}{\partial \sigma_{1, i k}} \\
\sigma_{2, i k}(t+1)=\sigma_{2, i k}(t)-\gamma \frac{\partial E}{\partial \sigma_{2, i k}} \\
\pi_{c, i k}(t+1)=\pi_{c, i k}(t)-\gamma \frac{\delta E}{\partial \pi_{c, i k}} \\
\pi_{v a r, i k}(t+1)=\pi_{v a r, i k}(t)-\gamma \frac{\delta E}{\partial \pi_{v a r, i k}}
\end{gathered}
$$

where $\gamma$ is the learning rate(step size) that must be carefully chosen as a large value may lead to instability, and small value on the other hand may lead to a slow learning process. The learning rate and IF-indexes for this study are fixed. Their adaptations will be considered in a future work. The derivatives in Equations (6) and (7) are computed as follows:

$$
\begin{aligned}
& \frac{\partial E}{\partial w_{i k}}=\frac{\partial E}{\partial y} \frac{\partial y}{\partial y_{k}} \frac{\partial y_{k}}{\partial w_{i k}}=\sum_{k} \frac{\partial E}{\partial y}\left[\frac{\partial y}{\partial y_{k}^{\mu}} \frac{\partial y_{k}^{\mu}}{\partial w_{i k}}+\frac{\partial y}{\partial y_{k}^{\nu}} \frac{\partial y_{k}^{\nu}}{\partial w_{i k}}\right] \\
& =\left(y(t)-y^{a}(t)\right) * \\
& {\left[(1-\beta)\left(\frac{\underline{f_{k}^{\mu}}}{\sum_{k=1}^{M} \underline{f}_{k}^{\mu}+\sum_{k=1}^{M} \bar{f}_{k}^{\mu}}+\frac{\bar{f}_{k}^{\mu}}{\sum_{k=1}^{M} \underline{f}_{k}^{\mu}+\sum_{k=1}^{M} \bar{f}_{k}^{\mu}}\right)\right.} \\
& \left.+\beta\left(\frac{\underline{f_{k}^{v}}}{\sum_{k=1}^{M} \underline{f_{k}^{v}}+\sum_{k=1}^{M} \overline{f_{k}^{v}}}+\frac{\overline{f_{k}^{v}}}{\sum_{k=1}^{M} \underline{f_{k}^{v}}+\sum_{k=1}^{M} \overline{f_{k}^{v}}}\right)\right] * x_{i} \\
& \frac{\partial E}{\partial b_{k}}=\frac{\partial E}{\partial y} \frac{\partial y}{\partial y_{k}} \frac{\partial y_{k}}{\partial b_{k}}=\sum_{k} \frac{\partial E}{\partial y}\left[\frac{\partial y}{\partial y_{k}^{\mu}} \frac{\partial y_{k}^{\mu}}{\partial b_{k}}+\frac{\partial y}{\partial y_{k}^{\nu}} \frac{\partial y_{k}^{\nu}}{\partial b_{k}}\right] \\
& =\left(y(t)-y^{a}(t)\right) *
\end{aligned}
$$

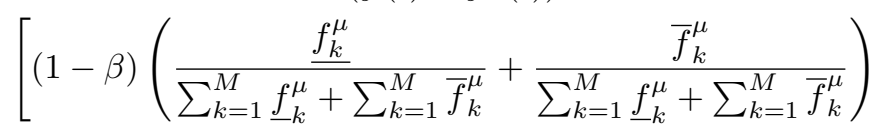

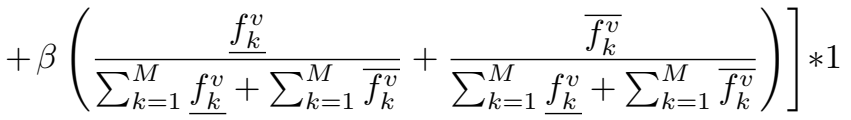

where $y_{k}$ is defined as in Equation (4). The derivatives in Equations (8) to (12) are computed as follows:

$$
\begin{aligned}
\frac{\partial E}{c_{i k}}=\sum_{k} \frac{\partial E}{\partial y}[ & \frac{\partial y}{\partial \underline{f}_{k}^{\mu}} \frac{\partial \underline{f}_{k}^{\mu}}{\partial \underline{\mu}_{i k}} \frac{\partial \underline{\mu}_{i k}}{\partial c_{i k}}+\frac{\partial y}{\partial \bar{f}_{k}^{\mu}} \frac{\partial \bar{f}_{k}^{\mu}}{\partial \bar{\mu}_{i k}} \frac{\partial \bar{\mu}_{i k}}{\partial c_{i k}} \\
& \left.+\frac{\partial y}{\partial \underline{f}_{k}^{\nu}} \frac{\partial \underline{f}_{k}^{\nu}}{\partial \underline{\nu}_{i k}} \frac{\partial \underline{\nu}_{i k}}{\partial c_{i k}}+\frac{\partial y}{\partial \bar{f}_{k}^{\nu}} \frac{\partial \bar{f}_{k}^{\nu}}{\partial \bar{\nu}_{i k}} \frac{\partial \bar{\nu}_{i k}}{\partial c_{i k}}\right]
\end{aligned}
$$

$$
\begin{gathered}
\frac{\partial E}{\sigma_{1, i k}}=\sum_{k} \frac{\partial E}{\partial y}\left[\frac{\partial y}{\partial \underline{f}_{k}^{\mu}} \frac{\partial \underline{f}_{k}^{\mu}}{\partial \underline{\mu}_{i k}} \frac{\partial \underline{\mu}_{i k}}{\partial \sigma_{1, i k}}+\frac{\partial y}{\partial \underline{f}_{k}^{\nu}} \frac{\partial \underline{f}_{k}^{\nu}}{\partial \underline{\nu}_{i k}} \frac{\partial \underline{\nu}_{i k}}{\partial \sigma_{2, i k}}\right] \\
\frac{\partial E}{\sigma_{2, i k}}=\sum_{k} \frac{\partial E}{\partial y}\left[\frac{\partial y}{\partial \bar{f}_{k}^{\mu}} \frac{\partial \bar{f}_{k}^{\mu}}{\partial \bar{\mu}_{i k}} \frac{\partial \bar{\mu}_{i k}}{\partial \sigma_{2, i k}}+\frac{\partial y}{\partial \bar{f}_{k}^{\nu}} \frac{\partial \bar{f}_{k}^{\nu}}{\partial \bar{\nu}_{i k}} \frac{\partial \bar{\nu}_{i k}}{\partial \sigma_{1, i k}}\right]
\end{gathered}
$$

where:

$$
\begin{aligned}
& \frac{\partial y}{\partial \underline{f_{k}^{\mu}}}=\frac{\partial y}{\partial \overline{f_{k}^{\mu}}} \\
&=(1-\beta)\left[\frac{y_{k}^{\mu}}{\sum_{k=1}^{M} \underline{f_{k}}+\sum_{k=1}^{M} \overline{f_{k}^{\mu}}}-\frac{y^{\mu}}{\sum_{k=1}^{M} \underline{f_{k}^{\mu}}+\sum_{k=1}^{M} \overline{f_{k}^{\mu}}}\right] \\
& y^{\mu}=\frac{\sum_{k=1}^{M}\left(\underline{f_{k}^{\mu}}+\overline{f_{k}^{\mu}}\right) * y_{k}^{\mu}}{\sum_{k=1}^{M} \underline{f_{k}^{\mu}}+\sum_{k=1}^{M} \overline{f_{k}^{\mu}}} \\
&=\beta\left[\frac{y_{k}^{\nu}}{\sum_{k=1}^{M} \underline{f_{k}^{v}}+\sum_{k=1}^{M} \overline{f_{k}^{v}}}-\frac{\frac{\partial y}{\partial f_{k}^{v}}}{\sum_{k=1}^{M} \underline{f_{k}^{v}}+\sum_{k=1}^{M} \overline{f_{k}^{v}}}\right] \\
& y^{\nu}=\frac{y_{k=1}^{\nu}}{\left.\sum_{k=1}^{M} \underline{f_{k}^{v}}+\overline{f_{k}^{v}}\right) * \sum_{k=1}^{\nu}}
\end{aligned}
$$

$\frac{\partial \underline{\mu}_{k}\left(x_{i}\right)}{\partial c_{i k}}=\left(1-\pi_{c, i k}\right) *\left(x_{i}-c_{i k}\right) * \exp \left(-\frac{\left(x_{i}-c_{i k}\right)^{2}}{2 * \sigma_{1, i k}^{2}}\right) / \sigma_{1, i k}^{2}$

$\frac{\partial \bar{\mu}_{k}\left(x_{i}\right)}{\partial c_{i k}}=\left(1-\pi_{c, i k}\right) *\left(x_{i}-c_{i k}\right) * \exp \left(-\frac{\left(x_{i}-c_{i k}\right)^{2}}{2 * \sigma_{2, i k}^{2}}\right) / \sigma_{2, i k}^{2}$

$\frac{\partial \underline{\mu}_{k}\left(x_{i}\right)}{\partial \sigma_{1, i k}}=\left(1-\pi_{c, i k}\right) *\left(x_{i}-c_{i k}\right)^{2} * \exp \left(-\frac{\left(x_{i}-c_{i k}\right)^{2}}{2 * \sigma_{1, i k}^{2}}\right)^{(14)} / \sigma_{1, i k}^{3}$

$$
\frac{\partial \underline{\mu}_{k}\left(x_{i}\right)}{\partial \sigma_{2, i k}}=\left(1-\pi_{c, i k}\right) *\left(x_{i}-c_{i k}\right)^{2} * \exp \left(\begin{array}{c}
\left.-\frac{\left(x_{i}-c_{i k}\right)^{2}}{2 * \sigma_{2, i k}^{2}}\right) \\
/ \sigma_{2, i k}^{3}(16)
\end{array}\right.
$$

The derivatives:

$$
\frac{\partial \underline{\nu}_{k}\left(x_{i}\right)}{\partial c_{i k}}, \frac{\partial \bar{\nu}_{k}\left(x_{i}\right)}{\partial c_{i k}}, \frac{\partial \underline{\nu}_{k}\left(x_{i}\right)}{\partial \sigma_{1, i k}}, \frac{\partial \bar{\nu}_{k}\left(x_{i}\right)}{\partial \sigma_{2, i k}}
$$

are obtained in the same manner as Equations (13) to (16). With the use of a t-norm prod operator,

$$
\begin{array}{rlrl}
\frac{\partial \underline{f_{k}}}{\partial \underline{\mu}_{i k}} & =\prod_{j=1, j \neq i}^{M 1} \underline{\mu}_{j k}, & \frac{\partial \overline{f_{k}}}{\partial \bar{\mu}_{i k}}=\prod_{j=1, j \neq i}^{M 1} \bar{\mu}_{j k} \\
\frac{\partial \underline{f_{k}^{v}}}{\partial \underline{\nu}_{i k}}=\prod_{j=1, j \neq i}^{M 1} \underline{\nu}_{j k}, & \frac{\partial \overline{f_{k}^{v}}}{\partial \bar{\nu}_{i k}}=\prod_{j=1, j \neq i}^{M 1} \bar{\nu}_{j k}
\end{array}
$$

The parameter $\beta$ in Equation (5), initially set at 0.5 , is learned to allow for adaptive adjustment of the membership and non- 
membership in the final output. The value of $\beta$ is adjusted as follows:

$$
\begin{aligned}
\beta(t+1)=\beta(t)-\gamma \frac{\partial E}{\partial \beta}, \quad \frac{\partial E}{\partial \beta}=\frac{\partial E}{\partial y} \frac{\partial y}{\partial \beta} \\
=\left(y-y^{a}\right)\left(\frac{\sum_{k=1}^{M}\left(\underline{f_{k}^{v}}+\overline{f_{k}^{v}}\right) * y_{k}^{v}}{\sum_{k=1}^{M} \underline{f_{k}^{v}+\sum_{k=1}^{M} \overline{f_{k}^{v}}}}\right. \\
\left.\quad-\frac{\sum_{k=1}^{M}\left(\underline{f_{k}^{\mu}}+\overline{f_{k}^{\mu}}\right) * y_{k}^{\mu}}{\sum_{k=1}^{M} \underline{f_{k}^{\mu}}+\sum_{k=1}^{M} \overline{f_{k}^{\mu}}}\right)
\end{aligned}
$$

Next, we present our experimental analysis and discussion of simulation results. The performance criterion used for all experiments is the root mean square error (RMSE) which is defined as:

$$
R M S E=\sqrt{\frac{1}{N} \sum_{i=1}^{N}\left(y^{a}-y\right)^{2}}
$$

where $y^{a}$ is the desired output and $y$ is the output of the model, $N$ is the number of testing data points.

\section{EXPERIMENTS AND RESULTS}

\section{A. Mackey Glass Prediction}

Mackey-Glass is a well known time series dataset defined by the following differential delay equation:

$$
\frac{d x(t)}{d t}=\frac{a * x(t-\tau)}{1+x(t-\tau)^{n}}-b * x(t)
$$

where $a, b$ and $n$ are constant real numbers, $t$ is the current time and $\tau$ is a non-negative time delay constant. The system tends to display a deterministic/periodic behaviour at $\tau \leq 17$ which turns chaotic when $\tau>17$. For comparison with other works in the literature such as [14], [20]-[23], where the target output is chosen as $x(t+6)$, from input vector $(x(t-18), x(t-12), x(t-6), x(t))$ and $\tau=17$. For each input in this study, two intuitionistic Gaussian membership functions with uncertain standard deviation are used. Similar to [22], the 1000 learning data are generated from time series $(\mathrm{t}=19$-1018) with the first 500 data points used for training and the remaining 500 for testing. The results of applying different approaches to the prediction of Mackey-Glass are listed in Table I. As shown in Table I, IT2IFLS outperforms the modified differential evolution radial basis function neural network (MDE-RBF NN) and the fuzzy approaches.

For a fair comparison with IT2FLS(TSK) [14], another experiment is conducted with the same computational settings, and number of training (1000) and testing (200) datasets as reported in [14]. The parameters of IT2FLS(TSK) are updated using GDA and equally used the same parameter $\beta$ to adjust the upper and lower values of the membership grades in the final output. As shown in Table II, after training, IT2IFLS outperforms IT2FLS(TSK) with the RMSE of 0.0168. We also implemented a T1 intuitionistic fuzzy logic system (IFLS) for Mackey-Glass prediction in order to evaluate the performance of the IT2IFLS over its T1 model. From Tables I and II, IT2IFLS outperforms IFLS because of the extra degrees of freedom offered by the FOUs of the IT2IFLSs.

TABLE I: Mackey-Glass Time Series Forecasting

\begin{tabular}{|c|c|c|}
\hline Models & train/chk & RMSE \\
\hline ANFIS Ensemble with IT2 FLS [24] & $400 / 400$ & 0.04933 \\
\hline ANFIS Ensemble with T1 FLS [24] & $400 / 400$ & 0.12043 \\
\hline Fuzzy-Singular Value Decomposition [22] & $500 / 500$ & 0.012 \\
\hline MDE-RBF NN [21] & $500 / 500$ & 0.013 \\
\hline Genetic Fuzzy Ensemble [23] & $500 / 500$ & 0.0264 \\
\hline Fuzzy Genetic Algorithm [23] & $500 / 500$ & 0.049 \\
\hline Radial Basis Function AFS [20] & $500 / 500$ & 0.0114 \\
\hline IFLS-TSK & $500 / 500$ & 0.0236 \\
\hline IT2IFLS-TSK & $500 / 500$ & 0.0079 \\
\hline
\end{tabular}

TABLE II: Comparison of IT2FLS(TSK) and IT2IFLS on Mackey-Glass Time Series

\begin{tabular}{|c|c|c|}
\hline Models & train/chk & RMSE \\
\hline IT2FLS(TSK) [14] & $1000 / 200$ & 0.0250 \\
\hline IFLS-TSK & $1000 / 200$ & 0.0234 \\
\hline IT2IFLS-TSK & $1000 / 200$ & 0.0168 \\
\hline
\end{tabular}

TABLE III: Non-linear System Identification

\begin{tabular}{|c|c|c|c|}
\hline Models & $\begin{array}{l}\text { Number } \\
\text { of } \\
\text { rules }\end{array}$ & $\begin{array}{l}\text { Number of } \\
\text { parameters }\end{array}$ & RMSE \\
\hline $\begin{array}{c}\text { T2FLS } \\
\text { (Singleton) [25] }\end{array}$ & 5 & 49 & 0.034 \\
\hline T2FLS (TSK) [25] & 3 & 36 & 0.0388 \\
\hline SEIT2FNN [25] & 3 & 36 & 0.0062 \\
\hline TSCIT2FNN [26] & 3 & 34 & 0.0084 \\
\hline eT2FIS [27] & 14 & 70 & 0.053 \\
\hline Type-2 TSK FNS [28] & 4 & 24 & 0.03239 \\
\hline Feedforward & 3 & 36 & 0.0281 \\
\hline Type-2 FNN [29] & 3 & 36 & 0.0241 \\
\hline SIT2FNN [29] & 3 & 32 & 0.0146 \\
\hline IFLS-TSK & 4 & 36 & 0.00522 \\
\hline IT2IFLS-TSK & 4 & &
\end{tabular}

\section{B. System Identification}

For further evaluation, IT2IFLS is applied to a non-linear system identification problem where the dataset is generated by the following differential equation:

$$
y(t+1)=\frac{y(t)}{1+y^{2}(t)}+u^{3}(t)
$$

The variables $u(t)$ and $y(t)$ are used as inputs while $y(t+1)$ is the desired output. Training samples are generated using $u(t)=\sin (2 \pi t / 100)$. We adopt similar computational set up in [25]-[29] with 500 epochs and 200 samples. Similar to IT2IFLS, the parameters of T2FLS (Singleton and TSK) 
are tuned using GDA. The results in Table III show that the IT2IFLS outperforms both forms of T2FLSs. Our approach is also compared with three evolving T2FLSs namely, self evolving interval type-2 fuzzy neural network (SEIT2FNN) utilising IT2FS in the antecedents and TSK interval type-1 set in the consequent, TSK-type-based self-evolving compensatory IT2FNN (TSCIT2FNN) which utilises IT2FS in the antecedent and a crisp linear model in the consequent and evolving type-2 neural fuzzy inference system (eT2FIS) with antecedent T2FS and Mamdani-type consequent. As shown in Table III, IT2IFLS exhibits a low level of RMSE over these evolving T2FLSs. In particular, the performance of IT2IFLS is compared with Type-2 TSK Fuzzy Neural System (Type-2 TSK FNS) [28], TSCIT2FNN [26] and SIT2FNN [29], which also utilised the parameter $\beta$ to adjust the contribution of upper and lower membership values in their final outputs. The results show a clear performance improvement of IT2IFLS over Type2 TSK FNS, TSCIT2FNN and SIT2FNN. We also constructed an IFLS in order to compare the performance of the IT2IFLS with its T1 model on system identification. From Table III, there is a significant performance improvement of IT2IFLS over IFLS on system identification.

\section{CONCLUSION}

In this study, an IT2IFLS-TSK approach to prediction is presented. The IT2IFLS can accommodate more imprecision thereby modelling imperfect and imprecise knowledge better than some IT2FLS. The key point in this design is in the IFindex which models the level of uncertainty of every element in each set. In future, we intend to learn the parameters of the IT2IFLS using Gaussian membership function with uncertain mean and also to train IT2IFLS using hybrid approach of GD and Kalman filter and to evaluate these on real world datasets. We also intend to apply IFS to general T2FLS.

\section{ACKNOWLEDGEMENT}

This research work is funded by the Government of Nigeria under the Tertiary Education Trust Fund (TETFund).

\section{REFERENCES}

[1] L. A. Zadeh, "Fuzzy sets," Information and control, vol. 8, no. 3, pp. 338-353, 1965.

[2] H. Pranevicius, T. Kraujalis, G. Budnikas, and V. Pilkauskas, "Fuzzy rule base generation using discretization of membership functions and neural network," in Information and Software Technologies. Springer, 2014, pp. 160-171.

[3] S. Chiu, "Extracting fuzzy rules from data for function approximation and pattern classification," Fuzzy Information Engineering: A Guided Tour of Applications. John Wiley\&Sons, 1997.

[4] J. M. Mendel, "Uncertain rule-based fuzzy logic system: introduction and new directions," 2001.

[5] L. A. Zadeh, "The concept of a linguistic variable and its application to approximate reasoning-i," Information Sciences, vol. 8, pp. 199-249, 1975.

[6] K. T. Atanassov, "Intuitionistic fuzzy sets," Fuzzy sets and Systems, vol. 20, no. 1, pp. 87-96, 1986.

[7] V. Olej and P. Hájek, "If-inference systems design for prediction of ozone time series: the case of pardubice micro-region," in Artificial Neural Networks-ICANN 2010. Springer, 2010, pp. 1-11.

[8] O. Castillo, A. Alanis, M. Garcia, and H. Arias, "An intuitionistic fuzzy system for time series analysis in plant monitoring and diagnosis," Applied Soft Computing, vol. 7, no. 4, pp. 1227-1233, 2007.
[9] K. Atanassov and G. Gargov, "Interval valued intuitionistic fuzzy sets," Fuzzy sets and systems, vol. 31, no. 3, pp. 343-349, 1989.

[10] H. A. Hagras, "A hierarchical type-2 fuzzy logic control architecture for autonomous mobile robots," IEEE Transactions on Fuzzy Systems, vol. 12, no. 4, pp. 524-539, 2004.

[11] J. M. Mendel, R. I. John, and F. Liu, "Interval type-2 fuzzy logic systems made simple," IEEE Transactions on Fuzzy Systems, vol. 14, no. 6, pp. 808-821, 2006.

[12] D. D. Nguyen, L. T. Ngo, and L. T. Pham, "Interval type-2 fuzzy cmeans clustering using intuitionistic fuzzy sets," in IEEE Third World Congress on Information and Communication Technologies (WICT), 2013, pp. 299-304.

[13] P. Hájek and V. Olej, "Intuitionistic fuzzy neural network: The case of credit scoring using text information," in Engineering Applications of Neural Networks. Springer, 2015, pp. 337-346.

[14] E. Kayacan and M. Khanesar, Fuzzy Neural Networks for Real Time Control Applications: Concepts, Modeling and Algorithms for Fast Learning. Elsevier Science, 2015. [Online]. Available: https://books.google.co.uk/books?id=8X4VBgAAQBAJ

[15] J.-S. R. Jang, "ANFIS: Adaptive-network-based fuzzy inference system," IEEE Transactions on Systems, Man and Cybernetics, vol. 23, no. 3, pp. 665-685, 1993.

[16] R. I. John and C. Czarnecki, "A type 2 adaptive fuzzy inferencing system," in IEEE International Conference on Systems, Man, and Cybernetics, 1998., vol. 2, pp. 2068-2073.

[17] M. A. Khanesar, E. Kayacan, M. Teshnehlab, and O. Kaynak, "Analysis of the noise reduction property of type-2 fuzzy logic systems using a novel type-2 membership function," IEEE Transactions on Systems, Man, and Cybernetics, Part B: Cybernetics, vol. 41, no. 5, pp. 13951406, 2011.

[18] P. Hajek and V. Olej, "Defuzzification methods in intuitionistic fuzzy inference systems of takagi-sugeno type: The case of corporate bankruptcy prediction," in 11th IEEE International Conference on Fuzzy Systems and Knowledge Discovery (FSKD), 2014, pp. 232-236.

[19] P. Hájek and V. Olej, "Adaptive intuitionistic fuzzy inference systems of takagi-sugeno type for regression problems," in Artificial Intelligence Applications and Innovations. Springer, 2012, pp. 206-216.

[20] K. B. Cho and B. H. Wang, "Radial basis function based adaptive fuzzy systems and their applications to system identification and prediction," Fuzzy sets and systems, vol. 83, no. 3, pp. 325-339, 1996.

[21] H. Dhahri and A. M. Alimi, "The modified differential evolution and the RBF (MDE-RBF) neural network for time series prediction," in IEEE International Joint Conference on Neural Networks (IJCNN), 2006., pp. 2938-2943.

[22] H. Gu and H. Wang, "Fuzzy prediction of chaotic time series based on singular value decomposition," Applied Mathematics and Computation, vol. 185 , no. 2, pp. 1171-1185, 2007.

[23] D. Kim and C. Kim, "Forecasting time series with genetic fuzzy predictor ensemble," IEEE Transactions on Fuzzy Systems, vol. 5, no. 4, pp. 523-535, 1997.

[24] J. Soto, P. Melin, and O. Castillo, "A new approach for time series prediction using ensembles of ANFIS models with interval type-2 and type-1 fuzzy integrators," in IEEE Conference on Computational Intelligence for Financial Engineering \& Economics (CIFEr), 2013, pp. $68-73$.

[25] C.-F. Juang and Y.-W. Tsao, "A self-evolving interval type-2 fuzzy neural network with online structure and parameter learning," IEEE Transactions on Fuzzy Systems, vol. 16, no. 6, pp. 1411-1424, 2008.

[26] Y.-Y. Lin, J.-Y. Chang, and C.-T. Lin, "A tsk-type-based self-evolving compensatory interval type-2 fuzzy neural network (TSCIT2FNN) and its applications," IEEE Transactions on Industrial Electronics, vol. 61, no. 1, pp. 447-459, 2014

[27] S. W. Tung, C. Quek, and C. Guan, "eT2FIS: an evolving type-2 neural fuzzy inference system," Information Sciences, vol. 220, pp. 124-148, 2013.

[28] R. H. Abiyev and O. Kaynak, "Type 2 fuzzy neural structure for identification and control of time-varying plants," IEEE Transactions on Industrial Electronics, vol. 57, no. 12, pp. 4147-4159, 2010.

[29] Y.-Y. Lin, S.-H. Liao, J.-Y. Chang, and C.-T. Lin, "Simplified interval type-2 fuzzy neural networks," IEEE Transactions on Neural Networks and Learning Systems, vol. 25, no. 5, pp. 959-969, 2014. 